LEADING ARTICLE

\title{
Fibrosis progression in chronic hepatitis $C$ virus infection
}

\author{
G W McCaughan, J George
}

Chronic hepatitis $C$ virus infection is typically characterised by slowly progressive hepatic fibrosis. However, it is recognised that some patients do not progress while others rapidly develop significant fibrosis. Here, we review studies that have assessed factors that could influence this rate of fibrotic progression.

See end of article for authors' affiliations

......................

Correspondence to:

Professor G W

McCaughan, AW Morrow

Gastroenterology and

Liver Centre, Centenary

Research Institute,

Missenden Rd, Royal

Prince Alfred Hospital,

Camperdown NSW 2050,

Australia; g.mccaughan@

centenary.usyd.edu.au

Accepted for publication 13 October 2003

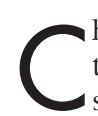
hronic hepatitis C virus (HCV) infection is typically characterised by slowly progressive hepatic fibrosis, with progression from stage 0 (no fibrosis) to stage 4 (cirrhosis) taking place at approximately $0.10-0.15$ fibrosis units (median) per decade. ${ }^{1}$ However, it is recognised that some patients do not progress while others rapidly develop significant fibrosis. Many studies have assessed the factors that could influence this rate of fibrotic progression. ${ }^{12}$ The best known variables are listed in table 1 . So what's new?

Although the variables listed in table 1 have been derived from multivariate analyses, several issues require consideration when interpreting these data. These include the nature of the reports, the suspicion that possible important pathogenic variables were not considered in the analyses, and thirdly that genetic predisposition to fibrotic progression may be crucial. Finally, do the published data have management and therapeutic implications? We now have some data to address these issues (table 2).

Firstly, with regard to the nature of the studies, most of the variables in table 1 were derived from cross sectional analysis. We now have more robust, prospective, longitudinal data with analysis of paired liver biopsy samples. In the paper by Ryder and colleagues ${ }^{3}$ in this issue of Gut, paired liver biopsies were analysed in 214 patients separated by a median of 2.4 years [see page 451 ]. Only $33 \%$ of patients showed fibrosis progression over time, with the two key predictors of progression being age at initial biopsy and presence of any fibrosis in the first biopsy. The latter suggests that fibrosis begets fibrosis. Neither of these concepts is new as cross sectional data also suggest that fibrosis is nonlinear over time, being more rapid at later stages ${ }^{4}$ In addition, cross sectional studies have indicated that age at acquisition of HCV infection is positively associated with the rate of fibrosis progression. ${ }^{2}$ Of particular interest, hepatic necroinflammation worsened in the second biopsy, suggesting that progressive inflammation may underlie the observed non-linear rates of fibrosis progression. On the other hand, the paper by Ryder et al is perhaps limited in that 183
Gut 2004;53:318-321. doi: 10.1136/gut.2003.026393

of 214 patients had very mild disease (stage $0 / 1$ ) at initial biopsy and the interval between biopsies was short, in relation to the long natural history of HCV infection. ${ }^{3}$ Despite these caveats, the data seem robust, and in light of the accumulating literature, real.

Another report with a similar design has also been published recently. ${ }^{5}$ In this smaller study $(n=123)$, patients had more fibrosis on the initial biopsy (mean 2.3 Ishak) and the interval between biopsies was greater (mean 44 months). Overall it was found that fibrosis progression was similar, with only 48 (39\%) patients demonstrating progression. In contrast with the report by Ryder and colleagues, ${ }^{3}$ the mean necroinflammatory score remained unaltered between the first and second biopsy. The rate of fibrosis progression was $\sim 0.12$ fibrosis units per year, and was higher in older patients and those with evidence of more necroinflammation in the initial biopsy. Interestingly, the rate of fibrosis progression was a quarter of that projected using the first biopsy and the estimated duration of infection data as the method of defining the rate of fibrosis progression. This contrasts with Poynard et al who found similar rates of fibrosis progression $(0.133 v 0.183)$ when these were estimated by either method. ${ }^{2}$ However, the latter data are not strictly comparable as fibrosis rates were calculated from different patient cohorts. A further prospective study $(n=76)$ of paired biopsies 3.7 years apart indicated that the initial activity grade was the single major determinant of fibrosis progression. ${ }^{6}$ Only $20 \%$ of patients had progression and these authors also suggested that rates of fibrosis evolution were lower when using paired biopsy rather than cross sectional analysis with the estimated duration of infection as the measurement tool. Similar but not identical findings were reported in a study of 98 patients in whom interface hepatitis was a key predictor of fibrosis progression more on the follow up than on the initial biopsy. ${ }^{7}$ These studies now set the benchmark for prospective analyses of the natural history of fibrosis evolution in chronic hepatitis $\mathrm{C}$ infection. The question remains however as to whether these studies are failing to take into account key pathobiological variables that could contribute to fibrosis progression in chronic hepatitis C infection.

Abbreviations: $\mathrm{HCV}$, hepatitis $\mathrm{C}$ virus; $\mathrm{HSC}$, hepatic stellate cell; $I R$, insulin resistance; $\mathrm{CHC}$, chronic hepatitis C; TNF, tumour necrosis factor; PPAR, peroxisome proliferator activated receptor; IFN- $\gamma$, interferon $\gamma$; TGF- $\beta$, transforming growth factor $\beta$ 
Table 1 Established factors associated with more advanced fibrosis in chronic hepatitis $\mathrm{C}$ virus (HCV) infection

- Male sex

- Duration of infection

- Acquisition of infection at $>40 y$

- Long term excessive alcohol consumption

- Long term immunosuppression (HIV, organ transplantation)

- HBV coinfection

- Non response to antiviral therapy

HIV, human immunodeficiency virus; HBV, hepatitis B virus.

"Hepatic inflammation appears to be the key pathological substrate that drives fibrogenesis"

As highlighted by all of these studies, hepatic inflammation appears to be the key pathological substrate that drives fibrogenesis. This makes sense as inflammation and cellular damage are intuitively linked to the fibrotic process via leucocyte/hepatic stellate cell (HSC) interaction, cytokine networks, proinflammatory small molecules (for example, reactive oxygen species), and insoluble mediators such as the hepatic neomatrix during wound healing. ${ }^{8}$ Two further reports have examined this link and the role of hepatic steatosis. ${ }^{10}$ Asselah et al undertook a prospective cross sectional analysis of 290 individuals with chronic hepatitis $\mathrm{C}$ infection. Higher levels of fibrosis were correlated with age at infection (again!) and a higher grade of hepatic necroinflammation. " This study also examined the effect of steatosis on fibrosis; steatosis was associated with fibrosis on univariate analysis but was not included in the multivariate model. This report closely mirrors earlier data demonstrating that independent predictors of hepatic fibrosis were age, portal inflammation, alanine aminotransferase levels, and previous alcohol intake, but not the extent of hepatic steatosis. ${ }^{11}$

We believe that a recent paper published by our group adds clarity to the relationship between hepatic inflammation, steatosis, and the stage of fibrosis in chronic HCV infection. ${ }^{10}$ In 260 patients with chronic hepatitis C infection, we demonstrated that both the extent of hepatic fibrosis and more importantly the rate of fibrosis progression were associated with insulin resistance (IR), as assessed by the homeostasis model assessment. Importantly, in light of abundant published data that type 2 diabetes is more prevalent in those with chronic hepatitis $\mathrm{C}$ (CHC) infection, IR in our cohort was associated with portal but not lobular inflammation. This suggests that IR in CHC infection is closely linked to the hepatic inflammatory response against the virus. The relationship between hepatic steatosis, IR, and fibrosis may also be genotype specific. Thus it is well recognised that genotype 3 infection may cause hepatic steatosis by a direct viral cytopathic effect on the hepatocyte. ${ }^{12}{ }^{13}$ Our recent paper also suggests that genotype 3 infection is associated with a lower incidence of IR. It is well known that chronic HCV infection is associated with increased levels of tumour necrosis factor (TNF) in the liver and serum of patients, and TNF elevations are associated with IR, in part by interference with insulin signalling. ${ }^{14}{ }^{15}$ It is therefore intriguing to speculate that TNF may be the key molecular link between inflammation, steatosis, and fibrosis in chronic $\mathrm{HCV}$ infection. In addition, hyperinsulinaemia itself may be a key lever of hepatic fibrogenesis. Thus hyperinsulinaemia directly stimulates HSC proliferation and secretion of extracellular matrix constituents ${ }^{16}$ and upregulates connective tissue growth factor, a key profibrogenic cytokine. ${ }^{17}{ }^{18}$ This pathway may therefore become a rational therapeutic
Table 2 Risk factors for fibrosis progression in chronic hepatitis $\mathrm{C}$ virus (HCV) infection: additional data

Longitudinal studies

- The hepatic necroinflammatory response ${ }^{35-7}$

Pathogenetic studies

- Insulin resistance ${ }^{10}$

Genetic studies

- Gene polymorphisms in inflammatory, fibrotic, and oxidative stress pathways $^{27-32}$

antifibrotic target in HCV infection. In this regard, insulin sensitising peroxisome proliferator activated receptor (PPAR) gamma agonists (for example, rosiglitazone, pioglitazone) are already in clinical use for the treatment of type 2 diabetes. This class of agents, among their myriad other actions, also have potentially beneficial therapeutic effects that can be exploited in $\mathrm{CHC}$ infected patients, including reduction of liver fat and direct anti-inflammatory and antifibrotic properties on HSCs. ${ }^{19}$

"TNF may be the key molecular link between inflammation, steatosis, and fibrosis in chronic HCV infection"

To add further complexity to this puzzle, we now have evidence of genetic links to fibrotic progression in all forms of liver injury, including chronic HCV infection. ${ }^{20}$ These data emerging from the above discussion clearly indicate that we need to examine the mediators of inflammation, fibrosis, and IR. Chronic HCV disease is characterised at the molecular and cellular level by a Thl-like inflammatory response, together with macrophage and HSC activation..$^{21}$ The molecules driving this process include interferon $\gamma$ $($ IFN $-\gamma)$, platelet derived growth factor, transforming growth factor $\beta$ (TGF- $\beta$ ), and connective tissue growth factor, ${ }^{23}$ as well as key chemokines. ${ }^{24}$ Although IFN- $\gamma$ is antifibrotic, the proinflammatory effects of IFN $-\gamma$ predominate and increased IFN- $\gamma$ expression correlates with portal but not lobular inflammation and also with fibrosis stage. ${ }^{25}$ The complexity and redundancies inherent in the wound healing hepatic response also suggests that defining genetic predispositions to fibrosis progression may be difficult. Furthermore, the effects of genetic polymorphisms may interact with viral replication itself (for example, a high interleukin 10 producing phenotype may inhibit fibrosis but in concert with a low IFN $-\gamma$ producing phenotype may result in an increase in viral replication and further immune stimulation/inflammation and fibrosis). ${ }^{26}$ Clearly, the answer to understanding genetic predisposition to liver disease progression in CHC (or other liver diseases) lies in large phenotypically well defined cohorts of patients together with family trios to examine transmission disequilibrium.

However, we do have some data on the predisposition to hepatic fibrosis in HCV infection. Polymorphisms in the angiotensinogen as well as the TGF- $\beta$ locus have been linked to more advanced stages of hepatic fibrosis in $\mathrm{CHC}$ infection. ${ }^{27}$ More recently, a polymorphism in the microsomal epoxide hydrolase gene, an enzyme involved in oxidative stress generation, has been associated with worse fibrosis, ${ }^{28}$ while Wright et al found a similar link to a mutation in the factor $\mathrm{V}$ Leiden gene. ${ }^{29}$ Genetic polymorphisms in antigen processing systems have been linked to HCV progression. ${ }^{30}$ Recently, polymorphisms in key inflammatory mediators known to be upregulated in chronic $\mathrm{HCV}$, such as Rantes and monocyte chemotatic factor, ${ }^{23}$ have also been identified as correlates with more severe HCV disease. ${ }^{31} 32$ 


\section{"Polymorphisms in key inflammatory mediators known to be upregulated in chronic HCV have been identified as correlates with more severe HCV disease"}

What are we to make of these putative genetic associations? Firstly, we need some ground rules to interpret the data. Significant patient numbers are required (usually in the several hundreds for disease severity studies and in the thousands for frequency comparisons with a normal population). It is also important that ethnic variation between groups is not significantly different. To a large extent this can be dealt with using family trios but this may not be feasible in the usual studies of HCV cohorts where typical sample sizes are uncommonly $<100$ and are more typically in the 100-300 range. Indeed, it has been suggested that only $30 \%$ of reported genetic associations in the latter setting are real! ${ }^{33}$ Finally, the tendency not to report negative results also needs to be factored. Ultimately, to avoid false positive associations, a working hypothesis related to functional data is required, preferably combined with actual data, that confirms a particular polymorphism is associated with a change in protein function. Thus genetic associations predisposing to hepatic fibrosis, although intriguing, need to be interpreted with caution. We need analyses of functionally relevant genes in large patient groups and their effects need to be discerned in multivariate analyses that include all of the factors that are known to influence progression of the disease of interest.

Finally, is there any hope of non-specific antifibrotic therapies? There are solid data to indicate that a sustained virological response in $\mathrm{CHC}$ infection is associated with a halt in the hepatic fibrotic (wound healing) response, and if limited to non-end stage liver disease, to fibrosis resolution. ${ }^{34}$ However, many patients still do not respond to antiviral therapy, while access and tolerability remain issues. In this scenario, the emergence of potential anti fibrotic targets is most welcome. An intriguing finding was presented recently in chronic HCV infection post liver transplant. The angiotensin/renin system has been implicated in tissue fibrosis in various organs and experimental systems. ${ }^{35} \mathrm{~A}$ recent retrospective analysis of post liver transplant patients with chronic HCV infection indicated that those on angiotensin receptors blockers had less liver fibrosis post transplant than those not taking such medications. ${ }^{36}$ Such an intriguing result requires further scrutiny and prospective analyses with regard to the power of such an effect. Similarly, as discussed earlier, the role of PPAR gamma agonists in preventing or reversing established hepatic fibrosis is an area of intense research. ${ }^{19}$

\section{"Demographic and epidemiological factors are linked to fibrosis progression in chronic HCV infection"}

To conclude, demographic and epidemiological factors are linked to fibrosis progression in chronic HCV infection. However, the importance of the hepatic necroinflammatory response is emerging as a key driver of this process. An increase in our understanding of the molecular regulation of this response and of the genetic predisposition of the host response to an injurious stimuli are likely to be the key foundations on which new therapies may emerge.

\section{ACKNOWLEDGEMENTS}

Grant support was from NHMRC, NIH RFA/DK/98.017.

\footnotetext{
Authors' affiliations

G W McCaughan, NHMRC Clinical Centre of Research Excellence in Outcomes for Chronic Liver Disease, and AW Morrow Gastroenterology and Liver Centre, Centenary Research Institute, University of Sydney, Royal Prince Alfred Hospital, Sydney, Australia
}

J George, NHMRC Clinical Centre of Research Excellence in Outcomes for Chronic Liver Disease, and Storr Liver Unit, Millennium Research Centre, University of Sydney, Royal Prince Alfred Hospital, Sydney, Australia

\section{REFERENCES}

1 Marcellin P, Asselah T, Boyer N. Fibrosis and disease progression in hepatitis C. Hepatology 2002;36:S47-56

2 Poynard T, Bedossa P, Opolon P, et al. Natural history of liver fibrosis progression in patients with chronic hepatitis C. Lancet 1997;349:825-32.

3 Ryder SD. Progression of hepatic fibrosis in patients with hepatitis C: a prospective repeat liver biopsy study. Gut 2004;53:451-5.

4 Yano $M$, Kumada $H$, Kage $M$, et al. The long-term pathological evolution of chronic hepatitis C. Hepatology 1996;23:1334-40.

5 Ghany MG, Kleiner DE, Alter $\mathrm{H}$, et al. Progression of fibrosis in chronic hepatitis C. Gastroenterology 2003;124:97-104.

6 Fontaine $\mathrm{H}$, Nalpas B, Poulet B, et al. Hepatitis activity index is a key factor in determining the natural history of chronic hepatitis $C$. Hum Pathol 2001;32:904-9.

7 Lagging LM, Westin J, Svensson E, et al. Progression of fibrosis in untreated patients with hepatitis $C$ virus infection. Liver 2002;22:136-44.

8 Maher JJ. Interactions between hepatic stellate cells and the immune system. Semin Liver Dis 2001;21:417-26.

9 Asselah T, Boyer N, Guimont M-C, et al. Liver fibrosis is not associated with steatosis but with necroinflammation in French patients with chronic heaptitis C. Gut 2003;52:1638-43.

10 Hui JM, Sud A, Farrell GC, et al. Chronic hepatitis $C$ virus infection and insulin resistance: how do they interact, and what is the effect on hepatic fibrosis? Gastroenterology 2003;125:1695-1704.

11 Hui JM, Kench J, Farrell GC, et al. Genotype-specific mechanisms for hepatic steatosis in chronic hepatitis C infection. J Gastroenterol Hepatol 2002;17:873-81.

12 Kumar D, Farrell GC, Fung C, et al. Hepatitis $C$ virus genotype 3 is cytopathic to hepatocytes: Reversal of hepatic steatosis after sustained therapeutic response. Hepatology 2002;36:1266-72.

13 Adinolfi LE, Gambardella M, Andreana A, et al. Steatosis accelerates the progression of liver damage of chronic hepatitis $C$ patients and correlates with specific HCV genotype and visceral obesity. Hepatology 2001;33:1358-64.

14 Nelson DR, Lim HL, Marousis CG, et al. Activation of tumor necrosis factoralpha system in chronic hepatitis C virus infection. Dig Dis Sci, 1997; 42:2487-94.

15 Hotamisligil GS. The role of TNFalpha and TNF receptors in obesity and insulin resistance. J Intern Med 1999;245:621-5.

16 Svegliati-Baroni G, Ridolfi F, Di Sario A, et al. Insulin and insulin-like growth factor- 1 stimulate proliferation and type I collagen accumulation by human hepatic stellate cells: differential effects on signal transduction pathways. Hepatology 1999;29:1743-51.

17 Paradis V, Perlemuter G, Bonvoust F, et al. High glucose and hyperinsulinemia stimulate connective tissue growth factor expression: a potential mechanism involved in progression to fibrosis in nonalcoholic steatohepatitis. Hepatology 2001;34:738-44.

18 Paradis V, Dargere D, Vidaud M De, et al. Expression of connective tissue growth factor in experimental rat and human liver fibrosis. Hepatology 1999;30:968-76.

19 Marra F, Efsen E, Romanelli RG, et al. Ligands of peroxisome proliferatoractivated receptor gamma modulate profibrogenic and proinflammatory actions in hepatic stellate cells. Gastroenterology 2000; 1 19:466-78.

20 Bataller R, North KE, Brenner DA. Genetic polymorphisms and the progression of liver fibrosis: a critical appraisal. Hepatology 2003;37:493-503.

21 Baroni GS, Pastorelli A, Manzin A, et al. Hepatic stellate cell activation and liver fibrosis are associated with necroinflammatory injury and Th1-like response in chronic hepatitis C. Liver 1999;19:212-19.

22 McCaughan GW, Gorrell MD, Bishop GA, et al. Molecular pathogenesis of liver disease: an approach to hepatic inflammation, cirrhosis and liver transplant tolerance. Immunol Rev 2000;174:172-91.

23 Shackel NA, McGuinness PH, Abbott CA, et al. Insights into the pathobiology of hepatitis $C$ virus-associated cirrhosis: analysis of intrahepatic differential gene expression. Am J Pathol 2002;160:641-54.

24 Heydtmann M, Shields P, McCaughan G, et al. Cytokines and chemokines in the immune response to hepatitis $C$ infection. Curr Opin Infect Dis 2001;14:279-87.

25 Napoli J, Bishop GA, McGuinness PH, et al. Progressive liver injury in chronic hepatitis $C$ infection correlates with increased intrahepatic expression of Th1associated cytokines. Hepatology 1996;24:759-65.

26 Nelson D, Soldevila-Pico C, Abdelmalek M, et al. Long-term use of recombinant interleukin 10 (IL-10) in patients with advanced chronic hepatitis C: insight into immunopathogenesis. Hepatology $2001 ; 34$ (suppl):430A.

27 Powell EE, Edwards-Smith CJ, Hay JL, et al. Host genetic factors influence disease progression in chronic hepatitis C. Hepatology 2000;31:828-33.

28 Sonzogni L, Silvestri L, De Silvestri A, et al. Polymorphisms of microsomal epoxide hydrolase gene and severity of HCV-related liver disease. Hepatology 2002;36:195-201.

29 Wright M, Goldin R, Hellier S, et al. Factor V Leiden polymorphism and the rate of fibrosis development in chronic hepatitis $\mathrm{C}$ virus infection. Gut 2003;52:1206-10. 
30 Akuta N Chayama K, Suzuki F, et al. Risk factors of hepatitis C virus-related liver cirrhosis in young adults: positive family history of liver disease and transporter associated with antigen processing 2(TAP2)*0201 allele. J Med Virol 2001:64:109-16.

31 Promrat K, McDermott DH, Gonzalez CM, et al. Associations of chemokine system polymorphisms with clinical outcomes and treatment responses of chronic hepatitis C. Gastroenterology 2003;124:352-60

32 Muhlbaver M, Bosserhoff AK, Hartmann A, et al. A novel MCP-1 gene polymorphism is associated with hepatic MCP-1 expression and severity of HCV-related liver disease. Gastroenterology 2003;125:1085-93.
33 loannidis JP. Genetic associations: false or true? Trends Mol Med 2003;9:135-8.

34 Shiratori Y, Imazeki F, Moriyama M, et al. Histologic improvement of fibrosis in patients with hepatitis $C$ who have sustained response to interferon therapy. Ann Intern Med 2000;132:517-24.

35 Paizis G, Cooper ME, Schembri JM, et al. Up-regulation of components of the renin-angiotensin system in the bile duct-ligated rat liver. Gastroenterology 2002;123: 1667-76.

36 Rimola A, Guevara $G$, Londono $M$, et al. Beneficial effect of antihyertensive drugs interfering with angiotensin on fibrosis associated with $\mathrm{HCV}$ infection recurrence after liver transplantation. Liver Transplant 2003;9:A132. 